\title{
THE COMPUTER CODE FOR INVESTIGATION OF THE MULTIPACTOR DISCHARGE IN RF CAVITIES
}

\author{
L.V. Kravchuk, G.V. Romanov, S.G. Tarasov, V.V. Paramonov, \\ Institute for Nuclear Research RAS,117312, Moscow, Russia
}

Abstract

The special code has been developed for numerical simulation of the multipactor discharge during long-time INR activity in the development and construction of accelerating structures. The code simulates the secondary emission electron trajectories at different levels of rf field using real electromagnetic field distribution (calculated with modern 2D or 3D software) in the cavity that has a complicated boundary shape. Special implementations have been developed for phase stability investigations. The velocity spread of secondary electrons also may be considered. The results of the simulations were compared with experimental data, showing good coincidence.

In this report, the methods of simulations, particularities and realization of the code are described. The examples of applications for discharge simulations in accelerating cavities also are presented.

\section{INTRODUCTION}

The Multipactor Discharge (MD) is a wellknown phenomenon in the development and construction of accelerating cavities. The discharge takes place at a low level of the electric field and usually MD can be seen during rf conditioning of accelerating cavities. However, in some regions of the cavity MD may take place at the operating level of the electric field at accelerating gaps, deteriorating parameters of the cavity. Many papers are dedicated to the MD study. In some papers and books, [1, 2] general properties of discharge are considered. These general conclusions allow providing some estimations and preliminary predictions for simple cavities. But MD is very complicated phenomena depending on real distribution of electromagnetic field (which can not be described analytically for complicated shapes of modern accelerating cavities), the level of fields, the surface conditions and so on. A direct numerical simulation is mostly the only way to investigate the MD possibility in the given accelerating cavity and to develop such cavity modifications that allow avoiding this possibility.

\section{PROBLEM DESCRIPTION}

During previous activity and at present stage attention has been paid to the MD study in accelerating cavities that have the symmetry of rotation. In the cylindric coordinates system we directly solve the equation of motion for nonrelativistic electron in singleparticle approximation.

To simulate electron motion, the real distribution of the electromagnetic field should be calculated by specialized codes and stored in the file. The code uses calculated values of electric field components $\mathrm{E} z, \mathrm{E} r$ and magnetic field $\mathrm{H}_{\llcorner}$in the meshpoints. If fields values between meshpoints are needed, the fast method of interpolation with spline-technique is used. To integrate the equation of motion, the conservative scheme of discretization is used and forth order Adams-method is applied.

\section{CODE ABILITIES}

First, the cavity boundaries should be specified to simulate the electron's motion in the cavity. The cavity boundaries may be formed partly of straight lines and partly of circular ones.

The introduced language for the boundary description is the same as well known SUPERFISH code. Such standardization simplifies essentially the user's work. The distribution of the electromagnetic field also may be taken from SUPERFISH, and the specially developed editor allows using for this purpose practically any known codes. This editor is rather simple.

The code simulates the electron emission from the specified part of the cavity boundary and simulates the electron motion to impact with another part of the boundary. The calculation of the phase of the electron flight precedes to the sorting of trajectories, which allows to specify resonant ones. The conditions of resonant discharge require that the flight phase of electrons in the discharge must be equal to $2 \pi \mathrm{n}$ (n- integer) with respect to the rf field period $2 \pi$. 
The order of the discharge and the number of collisions should be specified. It allows considering resonant trajectories of different configurations (for example, single-point discharge).

Wide possibilities of the code allow to investigate both the electron motion from specified point, under the given field level and the defined emission phase, and allow considering for one electron to run from the part of the boundary within the ranges of rf field levels and emission phases.
Considering relations between the phases of the electron emission and impact, a conclusion may be done concerning to the phase stability for resonant trajectories [5]. The special algorithm has been developed for this purpose. A lot of attention was paid to develop a userfriendly interface of the code. The user can see at the screen both trajectories directly and a lot of another useful information concerning to zones of stable discharge.

The typical example of the multipactor computing for the omega-shaped accelerating cell is shown on Fig. 1
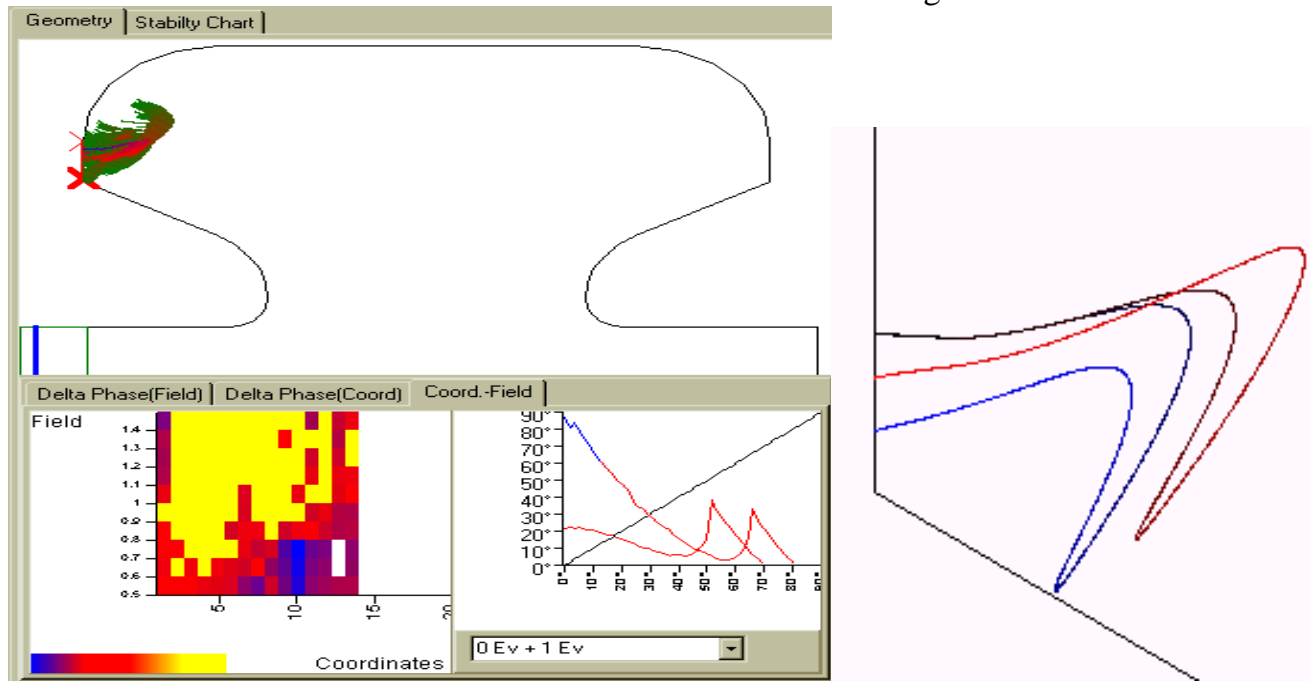

Fig. 1. Multipactor computing for the omega-shaped accelerating cell.

The present version of the code is developed under MS Windows-95 (NT) platform and may be run at any PC computer using this software. The control is totally mouse-driven.

\section{EXAMPLES OF APPLICATION}

The code was used for many applications in the development and construction of accelerating cavities at the INR. Historically first applications were for the MD study in the Disk and Washer accelerating structure during the construction of the high-energy part of the Moscow Meson Factory linac. Results of investigations were used for $\mathrm{rf}$ conditioning of DAW accelerating cavities.

The results of the MD investigations for PETRA cavities one can find in [3] For coupling cells of the Onaxis Coupled Structure (narrow cylindrical cavity, Fig.2) our investigations show the discharge excitation practically for all conditions of OCS applications [4].

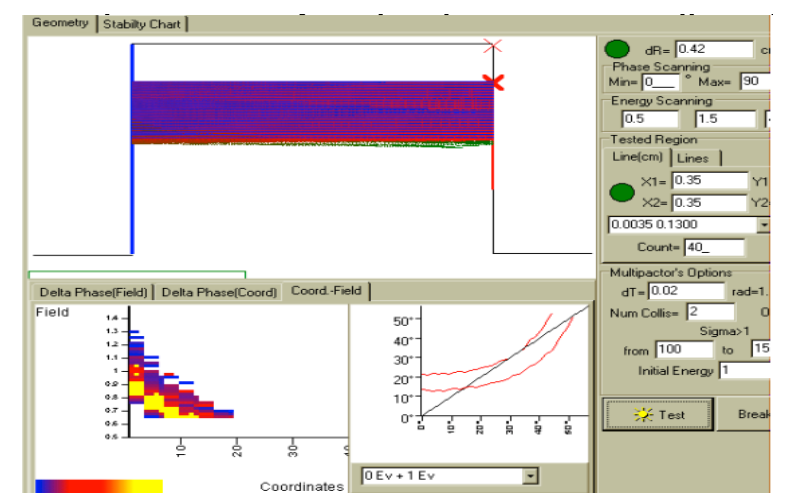

Fig. 2. Multipactor computing for coupling cells of the On-axis Coupled Structure. 
More complicated case takes place for coupling cells of the Side Coupled Structure (Fig.3). Usually this cell is formed having a central part like a capacitor. There are conditions for MD excitation in the central part of this capacitor. These conditions are simple enough if one considers electrons with zero emission energy and are more complicated if the real spread of emission energies is under consideration.

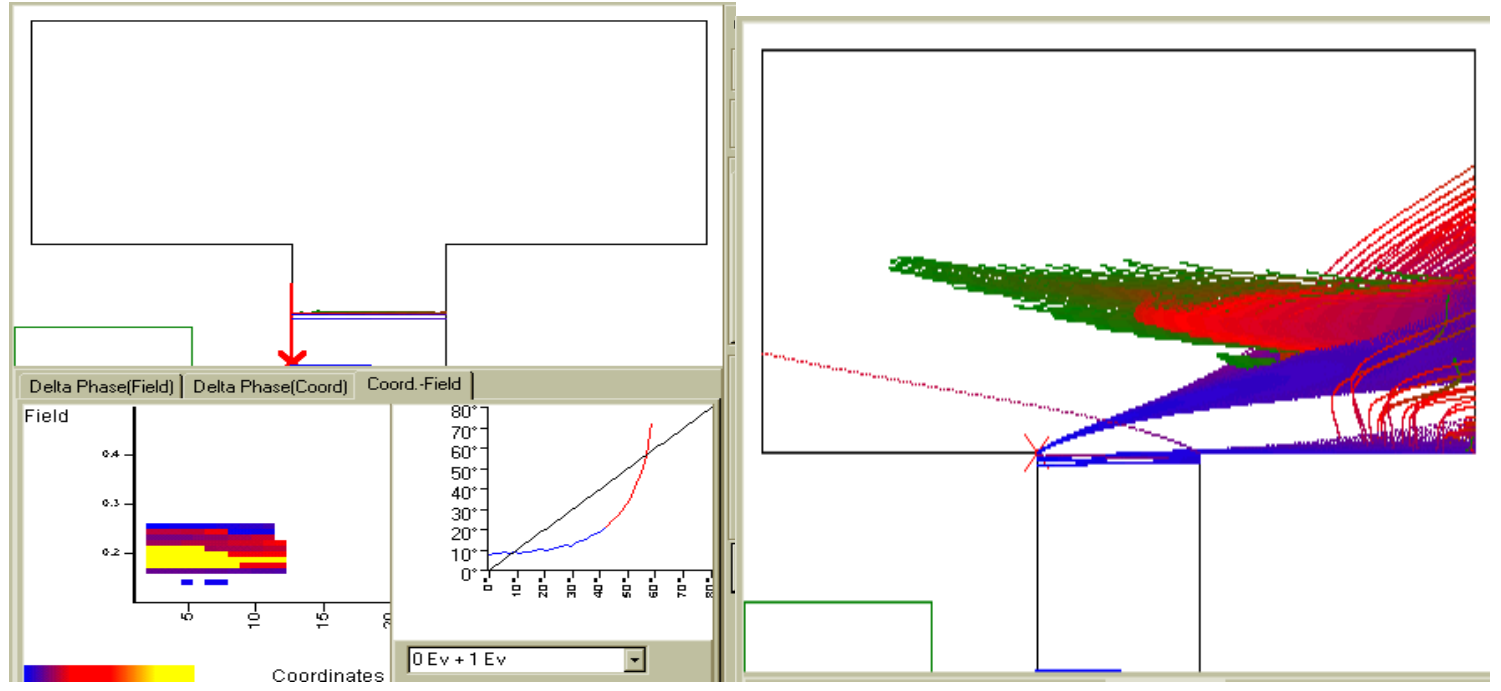

Fig. 3. Multipactor computing for coupling cells of the Side Coupled Structure.

Near outer radius of the capacitor, the non uniform electric field pumps out electrons (Fig.3), destroying conditions of the MD space stability [4].

\section{SUMMARY}

The described above code has been developed to investigate the multipactor phenomena taking into account real distributions of electromagnetic fields, real shapes of the cavity boundaries, different particularities of the discharge. Collected together in one powerful userfriendly code these possibilities allow to provide the investigation in conditions that are very close to the real ones, provide practical recommendations for the cavity shape modification, cavity conditioning and so on. The abilities of the code (and results obtained) exhibit it as an effective tool for investigations and an effective part of the design-kit for a cavity designer.

\section{REFERENCES}

[1] Slivkov I.N. Processes at high voltage in vacuum. Energoatomizdat, Moscow, 1986, (in Russian) and related references.

[2] D.Proch, D.Einfeld, R.Onken, Measurement of multi pacting currents... Proc. 1995 PAC Conf., p.1776-1778, 1995.

[3] I.Gonin, L.Kravchuk, G.Romanov et al. A study of multipactor phenomena in the $53 \mathrm{MHz}$ PETRA II cavity at DESY. Proc. of the 1994 EPAC, v.3, 2197, 1994.

[4] V. Paramonov, S. Tarasov, The possibility of multipactor discharge in coupling cells of coupled-cells accelerating structures. Proc. of the 1998 Linac Conference, Chicago, 1998 (to be published)

[5] V.A.Puntus, G.V.Romanov, Multipacting in the Phase Beam Monitor at MMF. Proc. of the 1998 EPAC, 1583, 1998 